meter) were made in the grafts to accept small piecies of iliac autografts in hope of bony renewal from them. Postoperatively they are closely observed for 11 and a half months, for 11 months, for 7 months and for 2 months respectively. Recent Xray examinations demonstrate secure coadunation of the grafts and the vaults. Active bony regenerations are in progress from the autogenous iliac bone chips packed in the trephine openings of the grafts.

\title{
33. Clinicopathological Study on Extracranial Metastases of Glioblastoma Multiforme
}

\author{
Kiyohide Komatsu, Hideo Hiratsuka, Shunpei Takahashi, \\ Akira Kamisasa, and Yutaka Inaba \\ Department of Neurosurgery, School of Medicine, \\ Tokyo Medical Dental University
}

Extracranial metastasis of glioblastoma multiforme has been said to be extremely rare. We experienced a case of glioblastoma multiforme with wide metastases to cervical lymph node, scalp, pericranium, spinal cord and cauda equina. We reported this case, and intended to review the literature of extracranial metastases of glioblastoma multiforme with respect to the frequency in sex and age, pathway and preference for sites of metastases, and the other clinicopathological pictures.

A 18 year-old female of right handed was admitted to our clinic with complaints of headache, vomiting and visual disturbance. Selective arterial-infusion of 5-FU and Mitomycin-C and irradiation were followed. Seven months after craniectomy left cervical lymph nodes enlarged to the size of pigeon-egg, biopsy of which revealed a malignant neurogenic neoplasm. The tumor extensively infiltrated through the scalp and pericranium to the left lateral neck, and subtotal removal of the tumor was performed, including the three fourth of the intracranial tumor mass, which weighed $788 \mathrm{~g}$. Immediate postoperative course was uneventful, but 10 days later, suddenly she went into coma after severe vomiting, and Froin's syndrome was noted by spinal tap. She expired 17 days after operation. At autopsy, the primary tumor was found in the left parieto-occipital region and metastasized widely to the left cervical lymph nodes, scalp, pericranium, dura mater, spinal subarachnoid space and cauda equina. Microscopical examination revealed that the tumor was glioblastoma multiforme. Such an extensive metastasis of glioblastoma multiforme as this seems very extremely rare. This case suggested some immunological aspects of glioma through CSF, considering the extremely rapid growing of the larger part of the primary tumor and its extracranial cervical extension.

Fifty-two cases of extracranial metastasis of glioblastoma multiforme were collected from the literatures. Of the total of 53 cases including our case, 42 (80\%) 
occurred in males and $11(20 \%)$ in females. The frequency of age was the type of normal distribution with the highest in the fifth decade. Sixty percent of the metastases resulted from the left hemisphere, because most of the tumor in the dominant hemisphere were given radiotherapy and/or chemotherapy after the decompressive craniectomy. Except two cases, all of them received various operations, and second or third operation was performed to 28 cases of them. There were two interesing cases of metastases through the ventriculo-pleural shunt. Radiotherapy were carried out to 30 cases $(57 \%)$ and chemotherapy to 5 cases $(9 \%)$. One year survival of metastatic cases was 25 , which was longer than a mean survival time of glioblastoma multiforme. The favered sites of metastases were, in order of frequency, cervical and mediastinal lymph nodes, lung, vertebral and the other bones, and liver. Also in the operative flap and the dural venous system, which were thought to be passages of all remote metastases, there were foci of metastases in 14 cases. It was possible to classify the pathway of metastasis of glioblastoma multiforme by following types;

1) Hematogenous metastasis through blood vessels of primary tumors.

2) Hematogenous metastasis through the dural vein invaded by tumor cells.

3) Hematogenous and/or lymphogenous metastasis from infiltrative foci in cranium, cranial soft tissues and the other tissues surrounding central nervous system.

4) Spread through cerebro-spinal fluid.

5) Hematogenous metastasis or dissemination via ventricular drainage tube. Type 1 of metastasis was scarcely present, and type 3 was seen in almost all of operative cases. Metastasis of non-operative cases was seen to be occurred by type 2 or type 4 . Type 5 was important, because in future such a type will be increases according as the therapy for malignant glioma would make progress.

\section{Classification of Thalamic Tumor by Vertebral Angiography}

Hiroshi Kuru, Itsuo Shiga, Hideshige Hayashi, Takaharu KawaI and Kazuo Hara

Department of Radiology, University of Osaka Department of Sumitomo Hospital 